\title{
PRECISION HOLE DRILLING USING A POLYCHROMATIC, BI-MODAL TECHNIQUE
}

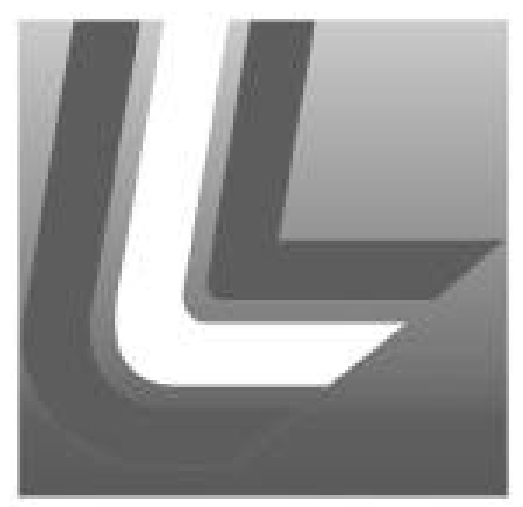

Lawrence Livermore National Laboratory

Making History Making a Difference

\section{2-2002}

Herb Friedman

Tracking Code: 01ERD099

UCRL-ID-146727

This work was performed under the auspices of the U.S. Department of Energy by the University of California, Lawrence Livermore National Laboratory under contract No. W-7405-Eng-48. 


\section{Background}

Rapid drilling of precision, submillimeter holes is needed for many industrial products including turbine blades, aircraft wings and fuel injectors. Recent results using ultra-short pulsed lasers shows promise but problems remain associated with poor beam quality, unexplained phenomena associated with deep holes and the difficult task of scaling these lasers to high average powers.

This effort makes use of results obtained with inexpensive Diode Pumped Solid State Lasers operating in the near infrared $(1064 \mathrm{n} \mathrm{m})$ and the second harmonic $(532 \mathrm{~nm})$ to drill precision submillimeter holes with arbitrary shapes in metals and ceramics. These lasers operate in the ablative regime (incident flux $=0.1-1 \mathrm{GW} / \mathrm{cm}^{2}$ ) with short pulse duration $(<100 \mathrm{~ns})$ to minimize the Heat Affected Zone. The high average power $(300 \mathrm{~W} @ 10 \mathrm{kHz})$ of these achieves drilling times of the order of a second or less in millimeter thick substrates. The thermal diffusion depth of these pulses of only a micron and the ablative process removes much of the incident power. By operating just above the ablative threshold and increasing throughput by increasing repetition rate (and therefore average power), harmful thermal effects are minimized.

The infrared beam, sized slightly smaller than the final hole diameter, rapidly removes the bulk of the material and allows an escape path for debris in the second step. The second step uses a much lower power, but near diffraction limited laser beam at the second harmonic wavelength. Laser coupling is better at the second harmonic wavelength that further reduces collateral thermal damage. This visible beam is focused to about $1 / 10$ the hole diameter and is used to clean, shape and polish the sides of the hole. With this "trepanning" laser, both shape and cross section can be cut to the desired dimensions while maintaining a minimum HAZ. 


\section{Results}

The experimental effort was started mid summer 2001 and concentrated on the reactivation of two surplus DPSSL's, one to operate at the fundamental wavelength for the basic hole drilling an the second at the second harmonic for the trepanning step. In the past, these lasers have produced over $500 \mathrm{~W}$ in the infrared and $300 \mathrm{~W}$ at the second harmonic and have demonstrated a Mean Time Between Failure of several thousand hours. The infrared (fundamental wavelength) laser has been optimized for beam quality and pulse duration to operate at $250 \mathrm{~W}, 13 \mathrm{kHz}$ and pulse duration of $85 \mathrm{~ns}$. Under these conditions, the laser flux over a $300 \mathrm{~m}$ diameter hole is $0.3 \mathrm{GW} / \mathrm{cm}^{2}$, just above the ablative threshold.

The infrared laser is shown in Fig. 1a with interlocks and controls. The optical train used to cut precision holes is shown in Fig. 1b. A view of the laser cutting holes in the millimeter substrate is shown in Fig. 2a and an end-on view showing a well rounded hole is given in Fig. $2 b$. A detailed examination of these holes shows that the HAZ is indeed negligible.

Figure $3 a$ is a metallographic cross section of a hole drilled by the infrared laser. There is a minimal amount of taper and some slag recast layer is evident on the sides of the hole. Metallographic acid etching of the cross section shows that the HAZ is negligibly small as indicated by the crystal boundaries that continue right up the sides of the hole. Figure $3 b$ is 200 times enlargement of the side of the hole in Fig. 3a and no disruption of the crystal boundaries are evident.

Work was also done to demonstrate the performance of the green trepanning laser in preparation for the next phase of the precision hole drilling demonstration. The second DPSSL was modified to include water cooled apertures which significantly improved the beam quality at the expense of power. For this laser application, the power needed to reach the ablation threshold is only of the order of $10 \mathrm{~W}$ since the laser spot diameter is reduced to 25 microns, i.e. 100 times less area than for the infrared beam.

The trepanning laser was operated at $20 \mathrm{~W}$ with a beam quality of 3-5 times diffraction limited and up to $50 \mathrm{~W}$ with a beam quality of 5-10 times diffraction limited. The $20 \mathrm{~W}$ performance level is sufficient for the trepanning operation and this laser will be used to demonstrate the next phase of the program. 


\section{Follow-on Program}

The experimental effort should continue to scope out the parameter space to optimize the basic hole drilling process using the infrared beam. Parameters to be studied include the effect on HAZ of peak power, pulse energy and beam shape. Diagnostic tools include beam analysis cameras to monitor beam shape incident on the substrate and metallographic sectioning/etching to measure the HAZ. Other issues for further study include:

- optimization of the cavity for operation in the infrared alone

- implementation of an electronic shutter to permit precise timing and/or pulse counting. This would to allow a thin membrane to be left intact at the bottom of the hole thereby minimizing the amount of energy penetrating the hole, an important criteria for industrial applications. The membrane would be removed by the low power trepanning laser at the conclusion of the hole drilling process.

- $\quad$ use of an Electro-Optic Q switch to permit shorter pulses, of the order of a few tens of nanoseconds, and therefore higher peak power. The advantage of this development would be either to drill larger diameter holes or reduce the lower PRF with a resultant decrease in average power. Such an E-O Q switch would need to handle high average power, of which present solid state devices are incapable. We propose to develop a new $Q$ switch based upon a flowing Kerr cell medium, a concept for which we have already filed a Record Of Invention.

- The experimental effort should also continue to demonstrate the effectiveness of the trepanning step. As a first step, the action of the trepanning can be demonstrated on a polished half plane. That is to say, the trepanning laser would be used to remove material on a flat, polished edge. The parameters of the trepanning step such as material removal rate and absence of a HAZ could be determined and there would be little or no issue of any recast layer since the ablated material has the entire half space to expand. The half space feature would also represent the action of the trepanning laser in a hole already drilled by the infrared laser. This is reasonable since the diameter of the trepanning laser is 10 times smaller than the hole diameter and to first order, the material ablated from the trepanning laser sees almost an entire half space to expand. These tests would be followed by the full dual beam, dual mode demonstration which involves a more complex optical arrangement. 




Fig. 1a. Diode Pumped Solid State Laser $u$ sed in the infrared for pilot Hole drilling studies.

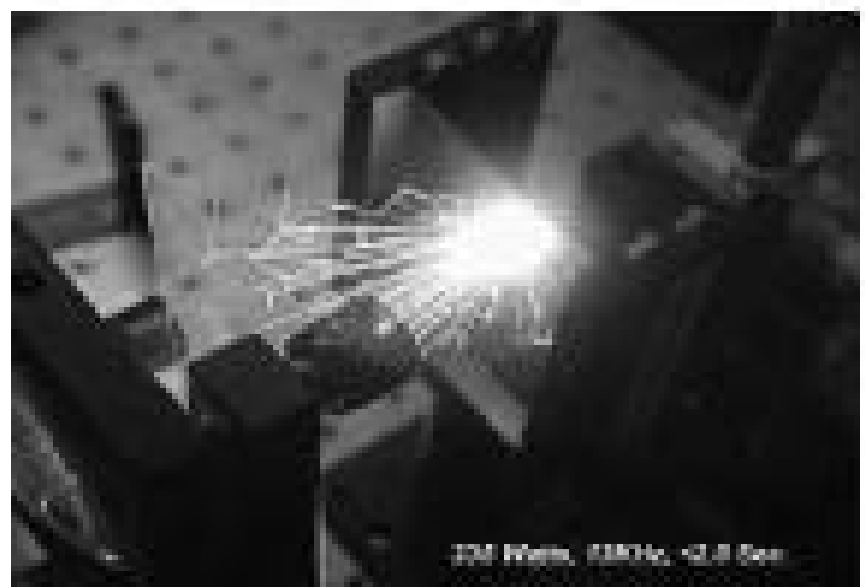

Fig. 2a. Infrared Laser Beam penetrating $1.5 \mathrm{~mm}$ of stainless steel for the pilot hole.



Fig. 1b. Optical train used in the the pilot hole drilling studies. The output beam from the DPSSL is collimated and then focused onto the metal target.



Fig. 2b. Microscope photo of the pilot hole showing the diameter of $1 / 4 \mathrm{~mm}$ and high precision of the radius.



Fig. 3a. 200 micron hole in $1.25 \mathrm{~mm}$ Stainless steel drilled with $235 \mathrm{~W}$ of $1064 \mathrm{~nm}$ pulsed power. Magnification is $50 x$.

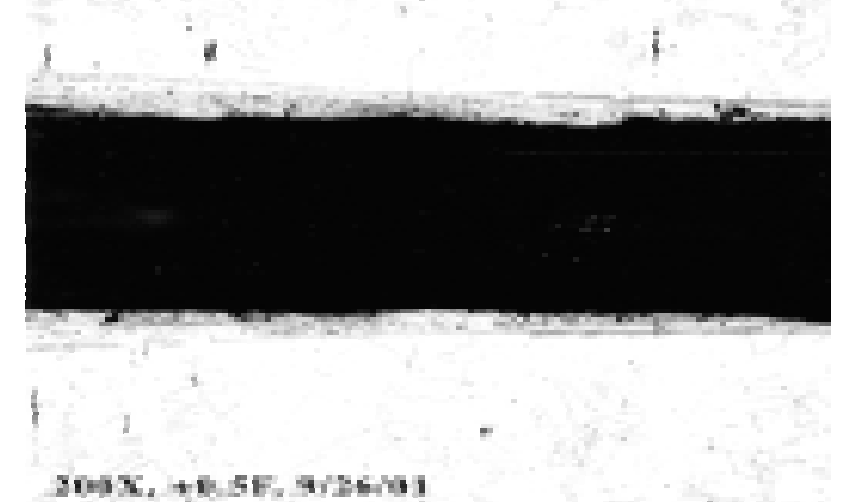

Fig. 3b. Same hole as Fig. 3a. With 200x magnification. Aside from the Zone is visible. 


\section{Summary}

It has been shown that the infrared beam with a pulse duration of $85 \mathrm{~ns}$ does not produce a measurable HAZ for submillimeter diameter holes in millimeter thick substrates, for incident peak fluxes just above the ablation threshold. This is in agreement with previous work performed in the visible and for shorter pulse durations. There us a thin recast layer and taper, both of which can easily be removed with the visible trepan laser. The speed of the infrared drilling process is rapid and the cost of the laser equipment is low (as compared to that of the ultrashort pulse laser approach) so that this two color, two modal approach appears to be attractive for many industrial applications. 